CentroSur

Social Science Journal

\section{Emociones, motivación y rendimiento académico: una propuesta para el desarrollo de habilidades orales en ingeniería desde la neuroeducación}

Research as the articulating axis of knowledge management in universities
Centro Sur.

Social Science Journal

Julio - Diciembre Vol 4 No 2

http://centrosureditorial.com/ind

ex.php/revista

eISSN: 2600-5743

revistacentrosur@gmail.com

Recepción: 8 marzo 2019

Aprobación 5 abril 2020

Pag 252- 265

Atribución/ReconocimientoNoComercial-Compartirlgual 4.0 Licencia Pública Internacional $\begin{array}{lll}\text { CC } & \text { BY-NC-SA } & 4.0 \\ \text { https://creativecommons.org/lice }\end{array}$ https://creativecommons.org/lice

Romina Oyarzún Yáñez
Gabriel Valdés-León²

\section{Resumen}

El objetivo de este trabajo es favorecer la motivación de estudiantes de ingeniería a través de una propuesta neurodidáctica con el fin de que logren aprendizajes significativos relacionados con las habilidades comunicativas orales. Para ello se diseñó un estudio de caso, con medición de pre y postest, que contó con la participación de 46 estudiantes de primer año de ingeniería quienes participaron de una unidad didáctica organizada en cinco sesiones con el fin de grabar un videocurrículum. A través de una encuesta ad-hoc que considera la motivación frente a tres aspectos (al curso, al desarrollo de la clase y al rol del docente), se recogen resultados que evidencian una relación estrecha entre la calificación obtenida y el desempeño académico en la tarea propuesta. Sobre esa base, se concluye que motivar a los estudiantes y propiciar un clima de aula positivo impactó de manera favorable en el rendimiento académico de los estudiantes.

Palabras lave: : neuroeducación, neurodidáctica, emociones, rendimiento académico, oralidad

1. Magister. Universidad Andrés Bello. Santiago, Chile. ORCID. https://orcid.org/0000-0001-9938-6019. Email. r.amaliaoyarzun@gmail.com. Google académico http://scholar.google.es/citations?user=DwqkaMAAAA AJ\&hl=es

2. Magister. Universidad Católica Silva Henríquez. Santiago, Chile. ORCID. https://orcid.org/0000-00018807-8838. Email.gvaldesl@ucsh.cl. Google Académico https://scholar.google.com/citations?hl=es\&user=wdbia IIAAAAJ 


\section{Abstract}

The purpose of this work is to encourage the motivation of students of engineering through a neurodidactic approach that leads to significant learning related to oral communication skills. A case study was designed for it, with pre and post measurement, which included the participation of 46 first-time engineering students who participated in a didactic unit organized in five sessions with the purpose of taking a video document. Through an ad-hoc survey that considers motivation in relation to three aspects (the course, the development of the class and the role of the teacher), results that demonstrate a strained relationship between the qualification obtained and the academic performance in it tarea propuesta. On this basis, it is concluded that motivating students and providing a positive classroom climate has a favorable impact on the academic performance of students.

Key words: neuroeducation, neurodidactic, emotions, academic performance, orality

\section{Introducción}

Dada la importancia que tiene el lenguaje, especialmente la comprensión lectora, la producción escrita y la comunicación oral, es fundamental potenciar las habilidades comunicativas para insertarse en la vida académica y, posteriormente, en el mundo laboral.

Las habilidades transversales (orales, escritas, de liderazgo, trabajo en equipo, empatía, entre otras) se desarrollan a través de la práctica y son esenciales para tener éxito profesional, pues resulta clave contar con las herramientas, las habilidades y la inteligencia emocional para trabajar de manera colaborativa y lograr un objetivo en común.

Sobre esta base, el trabajo que aquí se presenta tiene como objetivo es favorecer la motivación de estudiantes de ingeniería a través de una propuesta neurodidáctica con el fin de que logren aprendizajes significativos relacionados con las habilidades comunicativas orales. Así, nuestros esfuerzos se enmarcan en la alfabetización académica, pues buscamos potenciar "...nociones y estrategias necesarias para participar en la cultura discursiva de las disciplinas así como en las actividades de producción y análisis de textos requeridos para aprender en la universidad" (Carlino, 2003, p.410). El otro pilar sobre el cual se sustenta este trabajo es la neurodidáctica, pues cada una de las decisiones pedagógicas que permitieron diseñarlo e implementarlo han sido respaldadas en esta disciplina. Por ello, organizaremos este 
apartado de la siguiente manera: primero, daremos cuenta de la necesidad de potenciar las habilidades comunicativas en la educación superior y, específicamente, en la universidad en la que se realizó la investigación; luego, destacaremos los principales elementos de la neuroeducación presentes en la unidad didáctica (a saber, lenguaje, memoria y emoción); y, por último, describiremos los elementos medulares de dicha unidad.

La universidad en la que se llevó a cabo esta investigación es una institución chilena de financiamiento privado. Cuenta con más de 45.000 estudiantes (Emol, 2019) lo que la convierte en la casa de estudios superiores con mayor número de matriculados. Para ingresar a Ingeniería informática, carrera a la que pertenecen los estudiantes, es necesario obtener 450 puntos en la Prueba de Selección Universitaria (PSU), puntaje bastante bajo si consideramos que el máximo posible corresponde a 850 puntos.

Sumado a lo anterior, existe una desmotivación generalizada en relación con los cursos orientados hacia el desarrollo de competencias transversales que brinda la institución, desmotivación de la que ha sido testigo el docente a cargo del curso y que quedó en evidencia luego de la aplicación del pretest.

Tenemos, entonces, dos obstáculos que superar: por una parte, un conjunto de estudiantes universitarios con formación, intereses y habilidades bastante heterogéneas; por otra, un desinterés generalizado por aquellas actividades curriculares que no forman parte de la disciplina específica.

Para superar estas barreras, el énfasis de la propuesta estará en la motivación y, por lo tanto, en las emociones. Durante la implementación de la unidad se buscó motivar y conectar a los estudiantes con el curso, proponiéndoles actividades contextualizadas y vinculadas con su futuro desempeño profesional que les permitan adquirir habilidades comunicativas y de trabajo en equipo.

Como bien sabemos, la educación y, por lo tanto, el aprendizaje, es fundamental en la vida de una persona. Es por ello que los docentes tenemos la gran tarea de impactar positivamente en los alumnos para que no solo aprendan contenido necesario para la vida, sino que desarrollen habilidades y adquieran valores necesarios para desenvolverse en sociedad.

Activar conocimientos previos está directamente relacionado con la memoria (implícita, explícita y de trabajo), pero para acceder a ella es fundamental el componente emocional. El enfoque constructivista considera varios elementos que están vinculados con el cerebro límbico porque este enfoque señala que se deben 
activar conocimientos previos para construir un nuevo conocimiento, también, considera el lenguaje como una herramienta fundamental para que el docente medie el aprendizaje: activar conocimientos previos involucra la emoción, el lenguaje y la memoria: "'entendemos que el aprendizaje de un nuevo contenido es, en último término, el producto de una actividad constructivista que lleva acabo el alumno, mediante la cual construye e incorpora a su estructura mental los significados y representaciones relativos al nuevo contenido" (Coll, 1997, p.50).

El lenguaje es fundamental, es pensamiento hablado, nos permite razonar y buscar posibles soluciones a nuestros problemas, además, posibilita interactuar con los demás, establecer relaciones y las relaciones siempre son emocionales. A través de ellas podemos alcanzar aprendizajes, habilidades y valores para enfrentar la vida: "La conciencia emocional y la comprensión emocional pueden transformarse en el faro que nos ilumine el camino que lleva a la democracia, la paz y la felicidad, ya que la empatía es la base emocional de la democracia y sin ella, la sociedad no es sustentable" (Casassus, 2007, p.2).

Antiguamente, la inteligencia estaba relacionada exclusivamente a lo intelectual; hoy, además de la inteligencia intelectual, se reconoce también la inteligencia emocional, es decir, "utilizar la razón para gestionar las emociones" (Morgado, 2016). Somos los docentes quienes debemos enseñar a gestionar las emociones, ya que la pedagogía es una relación y las relaciones son emocionales. Para ello debe existir un compromiso emocional, esto marca la diferencia en la adquisición de aprendizajes.

Para tener una buena relación con los alumnos es importante crear un clima de aula positivo. Existen tres factores que nos permite lograrlo: el primero, la relación del docente con los alumnos; el segundo, las relaciones entre los alumnos y el tercero, el clima de paz o de tolerancia en la sala.

Tener un clima de aula tiene un impacto positivo en el aprendizaje de los estudiantes. Un estudio realizado por la UNESCO en 14 países de América Latina analizó los factores que afectan en el rendimiento académico y se identificó que el clima emocional del aula tiene un impacto en el rendimiento de los alumnos del $50 \%$ (Casassus, 2007). Esto se explica, entre otros aspectos, a causa de la capacidad que ciertas emociones tienen abrir o inhibir procesos de aprendizaje.

Cuando percibimos un estímulo consciente 0 inconsciente se ponen en funcionamiento "sistemas cerebrales como la atención, la motricidad, el sistema sensorial, los procesos cognitivos; activan el sistema vegetativo para dar información al cuerpo actuando en multitud de vísceras y estructuras vasculares y glandulares; activan el sistema endocrino para disponer de energía" (Casafont, 2012, p.16). Lo 
anterior plantea un desafío a los docentes y, sobre todo, a quienes trabajamos con grupos tan heterogéneos como los que se encuentran en la universidad en cuestión: distintas clases sociales, religiones diferentes, familias distintas y experiencias únicas. Estas características hacen que las motivaciones sean variadas. Por ejemplo, motivación por la calificación, por complacer a los padres o por complacer al profesor. La idea es que la motivación de los estudiantes sea intrínseca, es decir, que la implicación espontánea en una actividad les permita sentirse competente, autónomo y realizar la tarea porque lo disfrutan (American Psychological Association, 2015, p.18).

La gran diferencia de nuestro cerebro con otras especies es que posee la neocorteza $y$, en ella, la neocorteza prefrontal (CPF), área del lóbulo frontal, responsable de las funciones "que nos permiten elegir, planificar y tomar decisiones voluntarias y conscientes. Esta área está totalmente influida por la emoción. Su eficiencia se debe a su sensibilidad y a esa rápida repuesta ante la amenaza. La respuesta es rápida y automática y marca la diferencia entre la prioridad para ser atendido (Casafont, 2012). La memoria de trabajo, el lenguaje, el movimiento o la autoconciencia dependen del lóbulo frontal, así como la originalidad y la creatividad (para innovar hace falta conocer, lo que indica la importancia de la memoria de trabajo) (Guillén, 2012).

La propuesta didáctica consta de cinco clases, cada una de cuatro horas pedagógicas (180 minutos). El objetivo es que realicen presentaciones orales efectivas. Para ello, los estudiantes tendrán que realizar un videocurrículum considerando aspectos verbales, no verbales, paraverbales y la situación retórica. Asimismo, tendrán que entregar por escrito su discurso. A continuación, se presenta una síntesis de la planificación de la unidad propuesta (Tabla 1).

Tabla 1: Síntesis de las actividades de la propuesta didáctica

\begin{tabular}{l|l} 
Clase & Actividad de aprendizaje \\
\hline 1 & Inicio: la docente presenta a los estudiantes un tema \\
& controversial relacionado con su ámbito disciplinar. \\
& Desarrollo: luego, a partir del análisis de las \\
& opiniones de los propios estudiantes, presenta el \\
& modelo argumentativo ARE. \\
& Cierre: finalmente, la profesora los invita a \\
& estructurar sus opiniones a partir del modelo
\end{tabular}




\begin{tabular}{|c|c|}
\hline Clase & Actividad de aprendizaje \\
\hline & $\begin{array}{l}\text { propuesto para sintetizar los diferentes puntos de } \\
\text { vista. }\end{array}$ \\
\hline 2 & $\begin{array}{l}\text { Inicio: para comenzar, la docente les comenta a los } \\
\text { estudiantes que el segundo proyecto del curso } \\
\text { consiste en elaborar un videocurrículum. Luego, lee } \\
\text { una noticia sobre la importancia de las habilidades } \\
\text { comunicativas en las entrevistas laborales. Se } \\
\text { promueve la reflexión a través de preguntas. } \\
\text { Desarrollo: posteriormente, los invita a mirar un } \\
\text { video currículum. } \\
\text { Luego, la profesora formula las siguientes preguntas: } \\
\text { ¿Qué estructura tiene el discurso? ¿Qué aspectos } \\
\text { destaca? ¿Cuál es su formación académica? ¿Cuál } \\
\text { es su experiencia laboral? ¿Qué aspectos kinésicos } \\
\text { podemos destacar? } \\
\text { Una vez que hayan comprendido las características } \\
\text { del video currículum, la docente les comenta que } \\
\text { deben presentar un discurso argumentativo, ya que } \\
\text { busca convecer a un empledor. Para ello utilizarán el } \\
\text { modelo argumentativo ARE (afirmación, razón, } \\
\text { evidencia). }\end{array}$ \\
\hline
\end{tabular}

Cierre: finalmente, planifican discurso argumentativo de su videocurrículum aplicando el modelo ARE.

3

Inicio: los estudiantes observan varios videos con fragmentos de presentaciones orales. Luego, comentan en un plenario las diferencias entre estos y valoran la importancia de la comunicación verbal y no verbal.

Desarrollo: La docente guía la utilización de recursos no verbales y paraverbales en las presentaciones de los estudiantes. Luego, realizan una presentación oral de su currículum, considerando la importancia de una buena postura corporal y el uso de manos.

Cierre: Por último, la docente retroalimenta las presentaciones y entrega algunos consejos para 


\begin{tabular}{|c|c|}
\hline Clase & Actividad de aprendizaje \\
\hline & $\begin{array}{l}\text { impactar positivamente en la audiencia a través de } \\
\text { una buena postura corporal y el uso de manos } \\
\text { (evaluación formativa). }\end{array}$ \\
\hline 4 & $\begin{array}{l}\text { Inicio: para activar conocimientos previos, la } \\
\text { docente invita a los estudiantes a mirar un corto de } \\
\text { terror. Los estudiantes observan el video relacionado } \\
\text { con el miedo escénico y sus distintas } \\
\text { manifestaciones. En un plenario, comentan en qué } \\
\text { medida y de qué manera se ven afectados } \\
\text { cotidianamente. } \\
\text { Desarrollo: La docente presenta un PPT con } \\
\text { técnicas para enfrentar el miedo escénico. En } \\
\text { conjunto con el docente, se construye una lista con } \\
\text { algunas técnicas para superar el miedo escénico. } \\
\text { Cierre: Finalmente, los estudiantes preparan un } \\
\text { pequeño discurso y aplican aquellas que prefieran. }\end{array}$ \\
\hline 5 & $\begin{array}{l}\text { Inicio: los primeros } 20 \text { minutos de la clase, los } \\
\text { estudiantes se preparan para presentar su } \\
\text { videocurrículum. } \\
\text { Desarrollo: presentan su trabajo y son evaluados a } \\
\text { través de una rúbrica. } \\
\text { Cierre: la docente retroalimenta las presentaciones } \\
\text { orales y los estudiantes realizan comentarios de la } \\
\text { actividad. }\end{array}$ \\
\hline
\end{tabular}

La fundamentación neuroeducativa para cada una de las actividades tiene que ver con diferentes aspectos. En primer lugar, la selección de lecturas utilizadas en clases se realizó considerando textos vinculados con su carrera, lo que -desde la perspectiva de la alfabetización académica-, genera mayor motivación. Asimismo, durante las instancias de trabajo en aula, la docente guio la reflexión cautelando la relación entre lenguaje, la memoria y conocimientos. Por su parte, las instancias grupales favorecieron la interdependencia positiva y la responsabilidad individual y grupal. También, fomentó el uso adecuado de habilidades sociales. El aprendizaje cooperativo se traduce en un mayor rendimiento, mayor productividad, ayuda a mantener relaciones más comprometidas, solidarias (con ayuda de las neuronas 
espejo) traduciéndose en una mejor salud psicológica y una autoestima positiva (Margulis, 2016).

Otro aspecto que favorece la motivación es la utilización de textos multimodales (Martínez y Llorens, 2016). Al respecto, los estudiantes no solo revisaron y analizaron una muestra de videocurrículum, sino que tuvieron que elaborar uno como proyecto final del curso.

\section{Materiales y métodos}

En el marco de un diseño preexperimental, la investigación fue concebida como un estudio de caso, de tipo transversal y con un alcance descriptivo, en el que se aplicó un pre y postest.

Los participantes del estudio corresponden a 46 estudiantes de entre 18 y 20 años, vale decir, la totalidad de la cohorte 2019 para la carrera de Ingeniería informática de una universidad privada chilena. Cabe señalar que el curso inicialmente contaba con 52 alumnos, no obstante, seis de ellos no completaron alguno de los cuestionarios. La unidad didáctica se implementó en el marco de la actividad curricular Habilidades comunicativas, perteneciente al eje de formación general, el que se orienta hacia el desarrollo de habilidades transversales.

Como lo señalamos en $\$ 1.3$, el módulo didáctico consta de 5 sesiones, que corresponden a la segunda unidad del curso orientada hacia la competencia comunicativa oral. Progresivamente, se guio la reflexión desde la importancia de las habilidades comunicativas en el desarrollo académico y profesional hasta la elaboración de un producto que les permitió posicionarse como profesional en formación (ver imagen 1). Durante el desarrollo de las sesiones, se adoptó la orientación que ofrece Zabala (1999) respecto de la utilización de métodos globalizados de enseñanza: "[Los métodos globalizados] son métodos complejos de enseñanza, de manera explícita, que organizan los contenidos de aprendizajes a partir de situaciones, temas o acciones independientemente de la existencia o no de unas materias o disciplinas que hay que impartir" (p.24). 


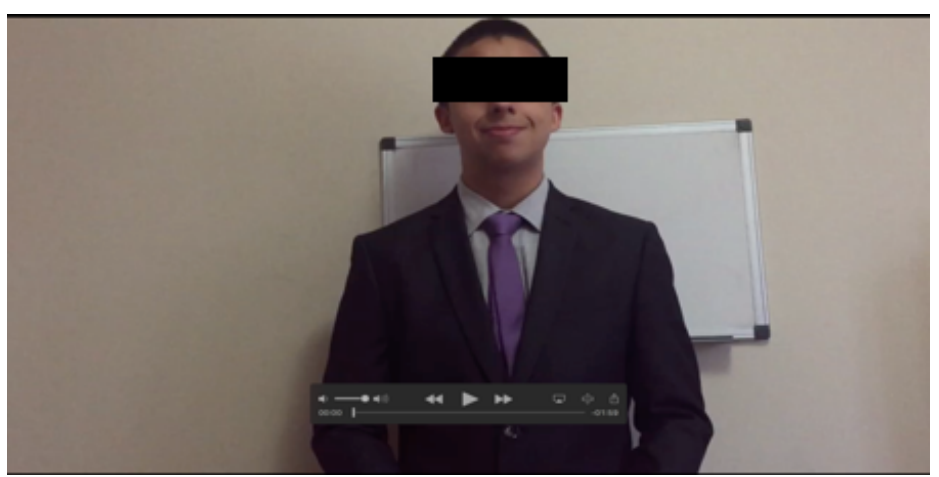

Imagen 1: Captura de video entregado por los estudiantes

Las variables consideradas en esta investigación son, por una parte, la motivación que manifestaron los estudiantes antes y después de la implementación del módulo didáctico y, por otra, el resultado académico en la tarea final de la unidad en cuestión $y$, por otra,

Para evaluar el desempeño comunicativo oral, se utilizó una rúbrica adaptada del trabajo de Valdés-León y Barrera (2020), instrumento que fue sometido al juicio experto de tres especialistas y, sobre esa base, ajustado al contexto particular de implementación. De manera sintética, podemos mencionar que los indicadores que conformaron la rúbrica fueron: organización textual, situación retórica, aspectos no verbales, aspectos paraverbales y diseño y uso del material de apoyo. Por su parte, la construcción del cuestionario sobre motivación se sustenta en una revisión bibliográfica que considera los trabajos de Manassero y Vásquez (1998), Roncel (2008), López Aguado y Falchetti (2009) y Navarro, Basanta y Abelairas (2017). Sobre esta base, se elaboró un cuestionario ad-hoc de 15 preguntas ítems en formato escala tipo Likert, en donde 1 equivale a 'totalmente en desacuerdo' y 5 a 'totalmente de acuerdo'. Se establecieron 3 categorías, a saber, curso, docencia y clase, y para cada una se establecieron cinco preguntas que indagaron en torno a la motivación para cada uno de estas.

Para el análisis de datos, se aplicó el instrumento para medir motivación antes de la implementación de la unidad didáctica a través de un cuestionario autoaplicado utilizando la plataforma Google Forms, mismo procedimiento que se realizó una vez terminada la unidad. Adicionalmente, se recogieron las calificaciones a través de una rúbrica y se realizó una comparación entre los resultados de motivación y el rendimiento académico obtenido utilizando Microsoft Excel como herramienta para procesar la información. 


\section{Resultados}

Los resultados obtenidos se presentarán como sigue: en primer lugar, se dará cuenta de los resultados totales considerando el promedio para el pre y postest en cada aspecto recogido en la encuesta, más la calificación promedio del curso; en segundo lugar, de forma particularizada, los resultados obtenidos por cada uno de los estudiantes (gráfico 1).

Como señalamos, esta tabla da cuenta de los resultados globales obtenidos por los estudiantes que participaron del estudio. Para su elaboración, se subdividieron los tres aspectos sobre los cuales se buscó conocer la motivación (el curso, la docencia y el desarrollo de la clase) de acuerdo con la información recogida en las instancias de pre y postest. Asimismo, se incorporaron los resultados académicos obtenidos luego de la unidad, dato que demuestra un rendimiento académico positivo.

No obstante lo positivo de los datos, es necesario reconocer que, dadas las características de la experiencia, no es posible atribuir los buenos resultados académicos única y exclusivamente al aumento de la motivación, pues muchos son los factores que inciden en el proceso educativo, ya sea internos o externos, y que pudieron incidir en ello. Sin embargo, a la luz de lo que se ha investigado sobre la relación entre motivación y rendimiento académico (entre otras, las investigaciones de Lamas (2008), Núñez (2009), Sánchez, (2016) y García-Ramírez (2016)) es viable pensar que la implementación de una propuesta didáctica respaldada en la neuroeducación y que, desde la propia voz de los participantes, favoreció la motivación, tuvo incidencia en estos resultados. Evidentemente, el objetivo del trabajo se aleja de un análisis pormenorizado de los resultados de cada uno de los estudiantes que participaron, sin embargo, el gráfico que aquí se expone (Gráfico 1) da cuenta de un aumento significativo en la motivación que manifestaron los estudiantes luego de la implementación. 


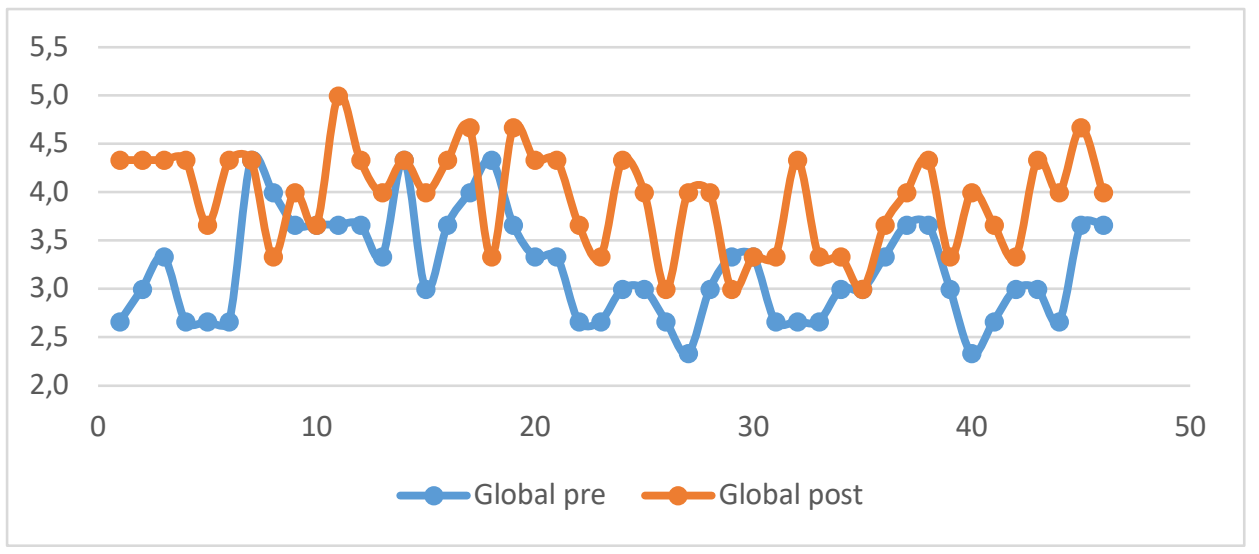

Gráfico 1: Resultados del pre y postest por estudiante.

Los casos de los estudiantes 7, 8, 28, 30 y 35, quienes mantuvieron o incluso disminuyeron su nivel de motivación, dan cuenta del carácter heterogéneo de la conformación de un curso y, como señalamos, pueden atribuirse a factores tanto internos como externos que quedan fuera del alcance de este trabajo. En ese sentido, consideramos que representa un número reducido de casos al compararlo con los estudiantes que mejoraron su nivel de motivación.

\section{Discusión}

El objetivo que nos propusimos en este trabajo fue motivar a los estudiantes de ingeniería que participaban del curso habilidades comunicativas a través de una propuesta neurodidáctica con el fin de que propiciar aprendizajes significativos respecto de las habilidades comunicativas orales. Al observar los resultados, podemos ver que, efectivamente, los estudiantes dan cuenta de un aumento en su motivación luego de haber desarrollado las actividades pedagógicas implicadas en la unidad, lo que puede relacionarse con los resultados favorables en su rendimiento académico. En este sentido, el trabajo hace eco de la importancia de considerar el funcionamiento del cerebro en el aprendizaje, especialmente, de las funciones ejecutivas y del cerebro emocional (límbico).

Para lograr esto, se tomaron decisiones pedagógicas fundamentales. Una de ellas tiene que ver con propiciar instancias que favorecieron la retroalimentación específica, explicativa y oportuna sobre cómo alcanzar los aprendizajes esperados, lo que garantizó la mediación del aprendizaje de los estudiantes y el apoyo en la gestión de sus emociones. Sobre esto último, los esfuerzos se orientaron hacia su racionalización, en primer lugar, para que pudieran tomar decisiones de manera 
consciente, utilizando el cerebro ejecutivo. Al respecto, Casafont (2012) releva la importancia de la inteligencia emocional: "La inteligencia no es cognición pura, sino que las variables cognitivas se yuxtaponen de modo inevitable con aspectos emocionales y motivacionales para generar una conducta inteligente" (p.120).

La segunda decisión pedagógica tiene que ver con la utilización de las emociones como un elemento esencial para el aprendizaje, dado su papel central en el aprendizaje: "la emoción básica, el interés, es la principal motivación para disponernos al comportamiento creativo, al bienestar y regular el sistema de percepción y atención" (Casafont, 2012, p.44). Los estudiantes se motivaron con las actividades propuestas, ya que fueron diseñads desde una perspectiva funcional y de manera contextualizada, lo que despertaba su interés y esto, a su vez, los movilizó. Asimismo, consideramos que se tomaron decisiones acertadas en cuanto a la activación de conocimientos previos al inicio de cada sesión, pues el énfasis estuvo en motivar y conectar a los estudiantes con las distintas actividades.

Como tercera decisión pedagógica, se buscó establecer una relación entre profesor y alumno cercana y respetuosa, de la misma forma que entre los mismos estudiantes. Esta decisión se justifica en la importancia que el clima de aula tiene en el aprendizaje, ya que, si los estudiantes no se sienten apoyados ni en confianza, no se atreverán a realizar presentaciones orales ni a trabajar de manera colaborativa. Al respecto, cabe señalar que todos los alumnos lograron presentar en público, retroalimentaron las presentaciones de sus compañeros y trabajaron de manera colaborativa.

\section{Conclusiones}

Como bien sabemos, el cerebro es sumamente importante en el aprendizaje. Cuando realizamos una acción, esta implica un registro en la corteza cerebral (Praxias y Gnosias). La conexión con el ambiente se realiza a través de los sentidos. A medida que los movimientos se repiten, se van consolidando, es decir, mientras más se practique, más rápidamente se aprenderá. Lo anterior justifica la cuarta y última decisión pedagógica adoptada: favorecer el 'aprender haciendo'. En esta propuesta, los alumnos tuvieron que desarrollar, clase a clase, desafíos que pusieron en juego su capacidad para resolver problemas, movilizando sus competencias comunicativas, con el fin de construir progresivamente su videocurrículum, un proyecto significativo, con una finalidad real y concreta que les permitió asumir su rol de ingenieros en formación. 


\section{Referencia}

American Psychological Association, Coalition for Psychology in Schools and Education. (2015). Top 20 principles from psychology for preK-12 teaching and learning. Disponible en http://www.apa. org/ed/schools/cpse/top-twentyprinciples.pdf

Carlino, P. (2003). Alfabetización académica: un cambio necesario, algunas alternativas posibles. Educere, 6(20), 409-420.

Casafont, R. (2012). Viaje a tu cerebro emocional . Grupo Zeta.

López Aguado, M., \& Falchetti, E. S. (2009). Estilos de aprendizaje. relación con motivación y estrategias. Revista De Estilos De Aprendizaje, 2(4). Recuperado a partir de http://revistaestilosdeaprendizaje.com/article/view/888

Casassus, J. (2007). El campo emocional en la educación: implicaciones para la formación del educador. Santiago de Chile: UMCE.

Coll, C. (1997). Constructivismo y educación escolar: ni hablamos siempre de lo mismo ni lo hacemos siempre desde la misma perspectiva epistemológica. El construcción del conocimiento escolar.

Emol. (21 de junio de 2019). UNAB, Autónoma y U. de Chile son las que más subieron su matrícula en 2019 y Universidad Inacap la que más cayó. El mercurio on line.

García-Ramirez, J. M. (2016). La motivación de logro mejora el rendimiento académico.

Guillén, J. (12 de agosto de 2012). Escuela con Cerebro . Recuperado el 30 de diciembre de 2019, desde https://escuelaconcerebro.wordpress.com/2012/08/12/el-lobulo-frontal-eldirector-ejecutivo-del-cerebro/

Lamas Rojas, H. (2008). Aprendizaje autorregulado, motivación y rendimiento académico. Liberabit, 14(14), 15-20.

Lirola, M. M., \& Simón, E. L. (2016). Aproximación al uso de recursos multimodales y de las TICs en la enseñanza del inglés. In XIV Jornadas de Redes de Investigación en Docencia Universitaria: investigación, innovación y enseñanza universitaria: enfoques pluridisciplinares (pp. 52-61). Instituto de Ciencias de la Educación. 
Manassero, M. y Vázquez, Ángel (1998). Validación de una escala de motivación de logro. Psicothema, 10(2), 333-351.

Margulis, L. (18 de agosto de 2016). Escuela con cerebro. Recuperado el 26 de enero de 2018, desde https://escuelaconcerebro.wordpress.com/2016/08/18/aprendizajecooperativo-y-neuroeducacion-guiando-la-poda-sinaptica/

Navarro Patón, R., Basanta Camiño, S., \& Abelairas Gómez, C. (2017). Los juegos cooperativos: incidencia en la motivación, necesidades psicológicas básicas y disfrute en Educación Primaria. Sportis, 3(3), 589-604.

Núñez, J. C. (2009, September). Motivación, aprendizaje y rendimiento académico. In Trabajo presentado en el X Congresso Internacional Galego-Português de Psicopedagogia. Braga, Portugal.

Sánchez, G. R. (2016). Atribución de motivación de logro y rendimiento académico en matemática. PsiqueMag, 4(1).

Valdés-León, G y Barrera, L. (2020) La reseña como puerta de entrada a los géneros académicos. Revista Formación Universitaria 13(4) [en prensa].

VEGA, V. M. R. (2008). Autoconcepto, motivación y ansiedad en el aula de idiomas. marcoELE. Revista de Didáctica Español Lengua Extranjera, (7), 1-20.

Zabala. A (1999) Enfoque globalizador y pensamiento complejo. Barcelona: GRAO 